\title{
A GLOBAL PINCHING THEOREM FOR COMPACT MINIMAL SURFACES IN $S^{3}$
}

\author{
YI-JUNG HSU
}

(Communicated by Jonathan M. Rosenberg)

\begin{abstract}
Let $M$ be a compact minimally immersed surface in the unit sphere $S^{3}$, and let $S$ denote the square of the length of the second fundamental form of $M$. We prove that if $\|S\|_{2} \leq 2 \sqrt{2} \pi$, then $M$ is either the equatorial sphere or the Clifford torus.
\end{abstract}

Let $M$ be a compact minimally immersed hypersurface in the unit sphere $S^{n+1}$. Denote by $S$ the square of the length of the second fundamental form of $M$. It is well known that if $0 \leq S \leq n$, then $M$ is either the equatorial sphere or a Clifford torus [1]. Recently, C. L. Shen [4, Theorem 2] proved that if $M$ is a compact embedded minimal surface of nonnegative Gauss curvature in the unit sphere $S^{3}$ with $\|S\|_{2}<1 /(6912 \sqrt{2 \pi(g+1)})$, then $M$ is the equatorial sphere, where $g$ denotes the genus of $M$. The purpose of this note is to improve this theorem and obtain the best constant. The following is our main result:

Theorem. Let $M$ be a compact minimally immersed surface in the unit sphere $S^{3}$. Then $\|S\|_{2} \geq 2 \sqrt{2 g} \pi$. The equality sign holds if and only if $M$ is either the equatorial sphere or the Clifford torus. In particular, if $\|S\|_{2} \leq 2 \sqrt{2} \pi$, then $M$ is either the equatorial sphere or the Clifford torus.

\section{NOTATIONS AND AUXILIARY RESULTS}

Let $M$ be a compact connected minimally immersed surface in the unit sphere $S^{3}$. Following the notations of [1], denote by $h=\left(h_{i j}\right)$ the second fundamental form of $M$, and by $S$ the square of the length of $h, S=\sum h_{i j}^{2}$. We need the following auxiliary results.

Lemma 1 [1]. $\frac{1}{2} \Delta S=S(2-S)+\sum h_{i j k}^{2}$, where $h_{i j k}$ denote the covariant derivatives of $h_{i j}$.

Received by the editors April 11, 1990.

1980 Mathematics Subject Classification (1985 Revision). Primary 53C42; Secondary 53C40. 
Lemma 2 [3]. The set of all zeros of $S$ is either the whole space $M$ or at most a finite set of points.

Lemma 3. $|\nabla S|^{2}=2 S \sum h_{i j k}^{2}$.

From Lemmas 1 and 3, we see that if $S$ is constant, then either $S \equiv 0$ or $S \equiv 2$.

Lemma 4. If $g \geq 1$, then

$$
\lim _{\varepsilon \rightarrow 0} \sum_{i=1}^{k} \int_{\partial B \varepsilon\left(p_{i}\right)} \frac{S_{r}}{S}=16 \pi(g-1)
$$

where $p_{1}, p_{2}, \ldots, p_{k}$ constitute all the zeros of $S$ and $S_{r}$ denotes the derivative of $S$ on $\partial B \varepsilon\left(p_{i}\right)$ in the radial direction from $p_{i}$.

Proof. At the points where $S$ is positive, by Lemma 3, we get

$$
\Delta \log S=2(2-S) .
$$

Integrating (1) over $M \varepsilon=M \backslash \bigcup_{i=1}^{k} B \varepsilon\left(p_{i}\right)$, we get from the Gauss equation

$$
2 K=2-S,
$$

where $K$ is the Gauss curvature of $M$, the assertion by Stokes's theorem and the theorem of Gauss-Bonnet.

\section{Lemma 5.}

$$
\int_{M} \sqrt{\frac{S}{2}}+\left(\frac{\pi}{4}-\sin ^{-1} \sqrt{\frac{S}{2+S}}\right) \frac{2-S}{2} \geq(g+1) \pi^{2} .
$$

Proof. Regard $M$ as an immersed surface of $\mathbf{R}^{4}$. Then the total absolute curvature of $M$, in the sense of [2], is given by

$$
\begin{aligned}
\int_{M} \int_{0}^{2 \pi}\left|(\sin \theta)^{2}-\frac{S}{2}(\cos \theta)^{2}\right| d \theta d V \\
\quad=\int_{M} 2 \sqrt{2 S}+\left(\pi-4 \sin ^{-1} \sqrt{\frac{S}{2+S}}\right) \frac{2-S}{2} .
\end{aligned}
$$

By a well-known inequality of Chern-Lashof [2], we have

$$
\int_{M} \sqrt{\frac{S}{2}}+\left(\frac{\pi}{4}-\sin ^{-1} \sqrt{\frac{S}{2+S}}\right) \frac{2-S}{2} \geq \frac{\pi^{2}}{2}\left(b_{0}+b_{1}+b_{2}\right),
$$

where $b_{i}$ denotes the $i$ th Betti number relative to the real field, for $i=0,1,2$. Since $M$ is of two-dimensional, $b_{0}=1, b_{1}=2 g$, and $b_{2}=1$. 


\section{Proof OF THEOREM}

We may assume that $S$ is positive except possibly at a finite set of points (Lemma 2). By using (1) and Lemmas 3 and 4, we get

$$
\begin{aligned}
\int_{M} 1+ & \frac{1}{4} h_{i j k}^{2}-\sqrt{\frac{S}{2}}-\left(\frac{\pi}{4}-\sin ^{-1} \sqrt{\frac{S}{2+S}}\right) \frac{2-S}{2} \\
= & \int_{M}\left[\frac{1}{\sqrt{2}(\sqrt{2}+\sqrt{S})}-\frac{1}{2}\left(\frac{\pi}{4}-\sin ^{-1} \sqrt{\frac{S}{2+S}}\right)\right](2-S)+\frac{1}{4} h_{i j k}^{2} \\
= & \lim _{\varepsilon \rightarrow 0} \int_{M \varepsilon} \frac{1}{2}\left[\frac{1}{\sqrt{2}(\sqrt{2}+\sqrt{S})}-\frac{1}{2}\left(\frac{\pi}{4}-\sin ^{-1} \sqrt{\frac{S}{2+S}}\right)\right] \Delta \log S+\frac{|\nabla S|^{2}}{8 S} \\
= & \lim _{\varepsilon \rightarrow 0} \int_{M \varepsilon} \frac{1}{2} \nabla\left[\frac{1}{2}\left(\frac{\pi}{4}-\sin ^{-1} \sqrt{\frac{S}{2+S}}\right)-\frac{1}{\sqrt{2}(\sqrt{2}+\sqrt{S})}\right] \nabla \log S+\frac{|\nabla S|^{2}}{8 S} \\
& -\lim _{\varepsilon \rightarrow 0} \int_{\partial M \varepsilon} \frac{1}{2}\left[\frac{1}{\sqrt{2}(\sqrt{2}+\sqrt{S})}-\frac{1}{2}\left(\frac{\pi}{4}-\sin ^{-1} \sqrt{\frac{S}{2+S}}\right)\right] \frac{S_{r}}{S} \\
= & -(4-\pi) \pi(g-1)+\lim _{\varepsilon \rightarrow 0} \int_{M \varepsilon}\left(\frac{1}{4}-\frac{1}{(2+S)(\sqrt{2}+\sqrt{S})^{2}}\right) \frac{|\nabla S|^{2}}{2 S} \\
= & -(4-\pi) \pi(g-1)+\int_{M}\left(\frac{1}{4}-\frac{1}{(2+S)(\sqrt{2}+\sqrt{S})^{2}}\right) h_{i j k}^{2} \\
\geq & -(4-\pi) \pi(g-1),
\end{aligned}
$$

where the equality sign holds if and only if $S$ is constant. According to Lemma 5 , we get

$$
\begin{aligned}
A+\frac{1}{4} \int_{M} h_{i j k}^{2} & \geq-(4-\pi) \pi(g-1)+\int_{M} \sqrt{\frac{S}{2}}+\left(\frac{\pi}{4}-\sin ^{-1} \sqrt{\frac{S}{2+S}}\right) \frac{2-S}{2} \\
& \geq 2 g \pi^{2}-4 \pi(g-1),
\end{aligned}
$$

where $A$ denotes the area of $M$. By combining (2) with the inequality (3), it follows that

$$
\int_{M} 2 S+h_{i j k}^{2}=4 A+16 \pi(g-1)+\int_{M} h_{i j k}^{2} \geq 8 g \pi^{2} .
$$

The desired inequality now follows from Lemma 1.

\section{REFERENCES}

1. S. S. Chern, M. Do Carmo, and S. Kobayashi, Minimal submanifolds of a sphere with second fundamental form of constant length, Functional Analysis and Related Fields, SpringerVerlag, 1970, pp. 59-75.

2. S. S. Chern and R. K. Lashof, On the total curvature of immersed manifolds. II, Michigan Math. J. 5 (1958), 5-12. 
3. H. B. Lawson, Complete minimal surfaces in $S^{3}$, Ann. of Math. 92 (1970), 335-374.

4. C. L. Shen, A global pinching theorem of minimal hypersurfaces in the sphere, Proc. Amer. Math. Soc. 105 (1989), 192-198.

Department of Applied Mathematics, National Chiao Tung University, Hsinchu, TaiWAN, RePublic OF China 